\title{
Observation of Zenneck-Like Waves over a Metasurface Designed for Launching HF Radar Surface Wave
}

\author{
Florent Jangal, ${ }^{1}$ Nicolas Bourey, ${ }^{2}$ Muriel Darces, ${ }^{2}$ François Issac, ${ }^{1}$ and Marc Hélier ${ }^{2}$ \\ ${ }^{1}$ ONERA-The French Aerospace Lab, 91123 Palaiseau Cedex, France \\ ${ }^{2}$ Sorbonne Universités, UPMC University of Paris 06, UR2, L2E, 75005 Paris, France \\ Correspondence should be addressed to Florent Jangal; florent.jangal@onera.fr
}

Received 4 May 2016; Revised 2 September 2016; Accepted 18 September 2016

Academic Editor: Weimin Huang

Copyright ( 2016 Florent Jangal et al. This is an open access article distributed under the Creative Commons Attribution License, which permits unrestricted use, distribution, and reproduction in any medium, provided the original work is properly cited.

\begin{abstract}
Since the beginning of the 20th century a controversy has been continuously revived about the existence of the Zenneck Wave. This wave is a theoretical solution of Maxwell's equations and might be propagated along the interface between the air and a dielectric medium. The expected weak attenuation at large distance explains the constant interest for this wave. Notably in the High Frequency band such a wave had been thought as a key point to reduce the high attenuation observed in High Frequency Surface Wave Radar. Despite many works on that topic and various experiments attempted during one century, there is still an alternation of statements between its existence and its nonexistence. We report here an experiment done during the optimisation of the transmitting antennas for Surface Wave Radars. Using an infrared method, we visualize a wave having the structure described by Zenneck above a metasurface located on a dielectric slab.
\end{abstract}

\section{Introduction}

In 1907, Zenneck was aiming to explain Marconi's experiments on transatlantic propagation [1]. Since ionization of the atmosphere was mainly thought as the result of Earth radioactivity and was limited to ten kilometers or so [2], Zenneck logically stated that the ionization of the atmosphere could not explain Marconi's results [1]. He assumed that transatlantic propagation was due to the creation of a new kind of electromagnetic wave which was propagated along the interface between the air and the ground. Zenneck claimed that this wave was a valid solution of Maxwell's equations and had the main features of a confined wave. Moreover it might have low attenuation along the interface. Two years later, Arnold Sommerfeld confirmed, with a more rigorous approach, that the Zenneck Wave (ZW) was a solution of the Maxwell equations [3]. The controversy then started since the mathematical solution proposed by Sommerfeld was questionable $[4,5]$. Among several arguments, a sign mistake was pointed out. This mistake led to the conclusion that the ZW was a calculus artefact and could not exist. Moreover, it froze the discussion in the mathematical domain and kept it aside the physical aspects $[6,7]$. As time goes by, according to mathematical approaches, an abundant terminology has been built to name the confined waves which can be propagated at the interface between two materials [8-12] and the ZW has remained a theoretical object. In this context, the main criticism is dealing with the excitation of the ZW. Indeed, the generation of a sole $\mathrm{ZW}$ requires a source of infinite dimension or a finite, but unphysical, phase lens. Those points could reinforce the idea that the $\mathrm{ZW}$ is a nonphysical solution $[13,14]$.

Thereby, many interpretations and beliefs have arisen about the ZW. Today, it is difficult to discern the way to provide a proof regarding its existence $[15,16]$ or nonexistence $[17,18]$. Nevertheless, the wave excited over an air/ground interface is a main issue for High Frequency (HF) Surface Wave Radars (SWR). The HFSWR can take advantage of low loss propagation along the interface. Hence, understanding the Zenneck Wave issue may allow improving the radar coverage by increasing the energy propagated along the ground.

It is well known that to deal with the complexity of wave excitation at air/ground interface we need to use negative 


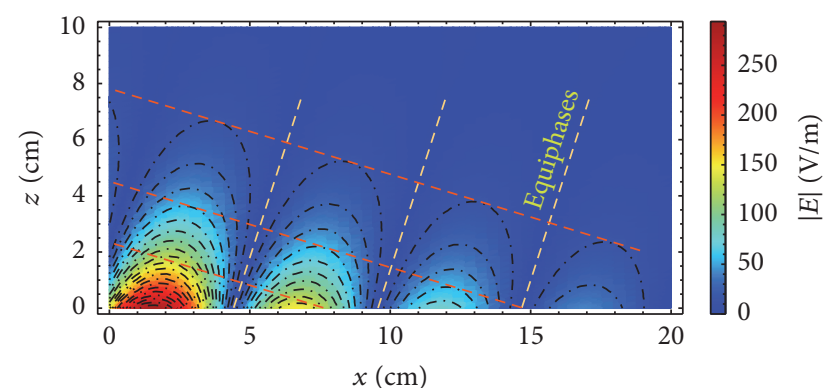

FIGURE 1: Simulation of a Zenneck Wave using Wait's formulation in [5]; the air/metasurface interface is located at $z=0$ and the relative permittivity is -1.2 .

permittivity and permeability materials [19]. This is the reason why we are using such materials.

In the next section we recall some theoretical aspects of the Zenneck approach. In Section 3 the measurement results are described. At last, Section 4 contains concluding remarks.

\section{Theoretical Approach}

2.1. The Zenneck Wave. More than one century ago, Zenneck himself has suggested some clues to better understand his assumption about the existence of a low loss Surface Wave. Nevertheless, he noticed that the phase speed of this wave exceeded the speed of the light. But, as recalled by Ling et al., the group velocity of the ZW along the interface never exceeds the speed of the light, whatever the phase velocity is $[1,20]$. Zenneck also remarked that the propagation vector was tilted toward the dielectric medium. As a result, the equiphase planes and the orthogonal equimagnitude planes are also slanted by comparison with the case of propagation above a perfect electric conductor $[21,22]$. The ZW can be represented using notably the formulation of James Wait [5] if a negative permittivity material is chosen for the ground as suggested in [19]. The excited wave is tilted towards the interface. Hence it seems to sink inside the lower dielectric medium (Figure 1). It also differs from the leaky waves by the tilt angle, since leaky waves are growing as they propagate and seem to rise away from the dielectric [19].

Apparently insignificant, that tilt can be one way to identify the ZW. We have to notice here, as shown in Figure 1, that the hypothetical weak attenuation suggested by Zenneck in order to explain Marconi's experiment is not valid since the attenuation is significant along the interface ( $x$ direction) and normally to the interface ( $z$ direction). This is in accordance with Hill and Wait conclusion: the ZW cannot be propagated over long distances [17]. Hence, ZW is not a good candidate to enhance HFSWR performances. But as shown hereafter, the understanding of the link between Surface Wave and Zenneck Wave allows designing Surface Wave launchers.

2.2. Zenneck Wave and Surface Plasmon Polariton. Considering the available theoretical studies [20, 23, 24], the physical reality of $\mathrm{ZW}$ and their ability to carry energy are still questionable. Those uncertainties are even more important

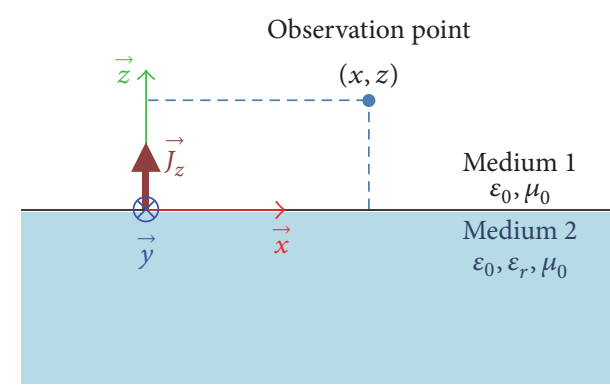

FIGURE 2: Geometry of the problem.

if we consider the doubts of Zenneck about his solution: the above-mentioned tilted equiphase planes violated the initial assumption of plane wave propagation. But, this discrepancy seems not to be a concern, at least for him.

To find a possible enhanced antenna for HFSWR, we proceed from the geometry depicted in Figure 2.

The upper medium is air; the lower medium is a lossy dielectric. The source is an infinite line of $\vec{z}$ oriented electric dipoles $\overrightarrow{J_{z}}$ lying along the $\vec{y}$-axis (magnitude of $\vec{J}_{z}$ is $1 \mathrm{~A} / \mathrm{m}^{2}$ ). The field at the observation point is the solution of Maxwell's equations obtained with modal decomposition [22]. For convenience, since we are focusing on the vertical electric field at $z=0$ and since the line source is located on the interface, the calculation is performed using the surface impedance approach [25-28] rather than with the development of the reflection coefficient at grazing angle [6]. Indeed, the impedance condition replaces the dielectric medium at $z=0$ and the vertical electric field is as follows [6]:

$$
\begin{aligned}
\left.E_{z}(x, z)\right|_{z=0} & =-\left.\widetilde{Z}_{s} \cdot H_{y}(x, z)\right|_{z=0}, \\
\widetilde{Z}_{s} & =\sqrt{\frac{\mu_{0}}{\varepsilon_{0}}} \cdot \sqrt{\frac{1}{\widetilde{\varepsilon}_{r}}}
\end{aligned}
$$

where $\left.E_{z}(x, z)\right|_{z=0}$ is the electric field normal to the interface; $\left.H_{y}(x, z)\right|_{z=0}$ is the magnetic field tangential to the interface; $\widetilde{Z}_{s}$ is the complex surface impedance of the dielectric; and $\widetilde{\varepsilon}_{r}$, $\varepsilon_{0}$, and $\mu_{0}$ are, respectively, the relative complex permittivity of the dielectric, the free space permittivity, and the free space permeability.

The magnetic field generated by the source can be formally expressed in an orthonormal basis consisting in a ZW (i.e., first term of (3)) and a continuous wave spectrum which can be viewed has spectral decomposition of other propagation modes (i.e., second term of $(3)$ ) [27, 28].

$$
\begin{aligned}
\left.H_{y}(x, z)\right|_{z=0}= & -i \cdot k_{z} \cdot e^{i \cdot \sqrt{k_{0}^{2}-k_{z}^{2}} \cdot x} \\
& +\frac{1}{\pi} \int_{0}^{\infty} \frac{p^{2} \cdot e^{i \cdot \sqrt{k_{0}^{2}-p^{2}} \cdot x}}{p^{2}-k_{z}^{2}} \cdot d p, \\
k_{z}= & -\widetilde{Z}_{s} \cdot \omega \cdot \varepsilon_{0},
\end{aligned}
$$

where $k_{z}$ is the complex wave number in the $z$ direction; $\omega$ is angular frequency; at last, the integrand $p \in \mathbb{R}$. 


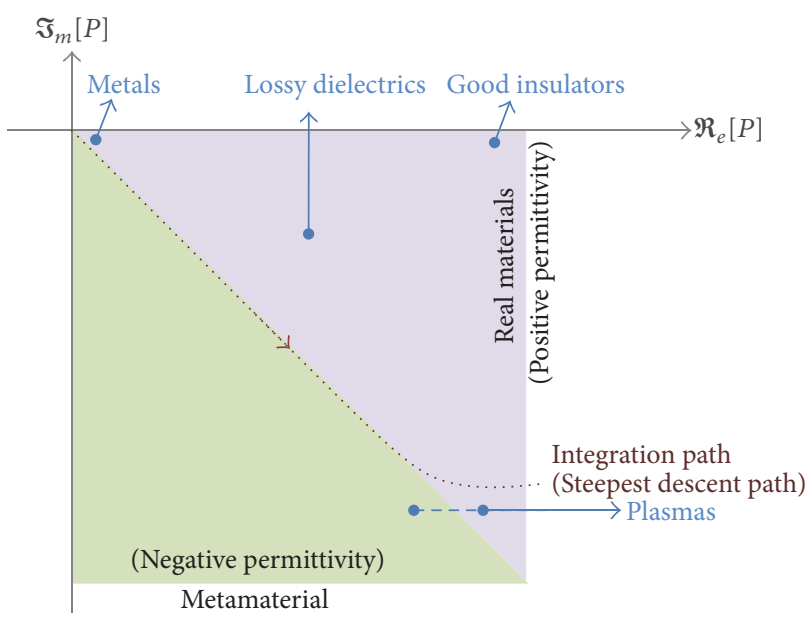

Figure 3: Position of pole $P$ in the complex plane according to the ground properties.

When computing the second part of (1), the pole $P=$ $-\widetilde{Z}_{s} \cdot \omega \cdot \varepsilon_{0}$ provides a contribution equal to the $\mathrm{ZW}$ term but with a positive sign. Thus, the $\mathrm{ZW}$ is not visible in the total field. We have here the mathematical core of the controversy: the ZW term disappears, even if it contributes to the final result. This is not surprising since this point has been largely discussed between Weyl and Sommerfeld early in the 20th century $[8,29]$ and many other authors. This point confirms the previous assumption of nonefficiency of $\mathrm{ZW}$ for long range propagation.

Nevertheless, as in other formulations [7, 9], the location of the pole $P=-\widetilde{Z}_{s} \cdot \omega \cdot \varepsilon_{0}$ with respect to the integration path determines the kind of the excited Surface Wave. The possible positions of $P$ in the complex plane are split near the origin by the steepest descent path. The upper right part corresponds to the usual materials including positive permittivity plasmas and the lower left part to the metamaterials (Figure 3).

The medium which can enhance radiation near the interface should have a pole not surrounded by the SDP. If we choose $\widetilde{Z}_{s}$ such that the pole does not contribute to the integral in (3), whatever the integrand $p$ is, the neutralization of the ZW will not occur.

An obvious way to do that is to insert an inductive surface over the dielectric. It has been shown by many authors in the course of the controversy that such a surface can propagate a confined wave traveling at the air-dielectric interface [24, $29,30]$. A metamaterial can provide the needed impedance and move the pole outside the integration path. In this case we will generate the well-known Surface Plasmon Polariton (SPP) which is propagated along an inductive surface $[31,32]$.

SPP has been extensively studied in optic domain [26, 33]. In optic wavelengths, the waves strongly interact with the free charges in metals [26]. As a result the medium is highly dispersive and the permittivity can reach negative values. In the same way, at radio frequencies, the dispersity of metamaterials allows reaching such negative values. Hence, it is possible at radio frequencies to generate a wave having the same behaviour as the SPP's one [32, 34, 35].

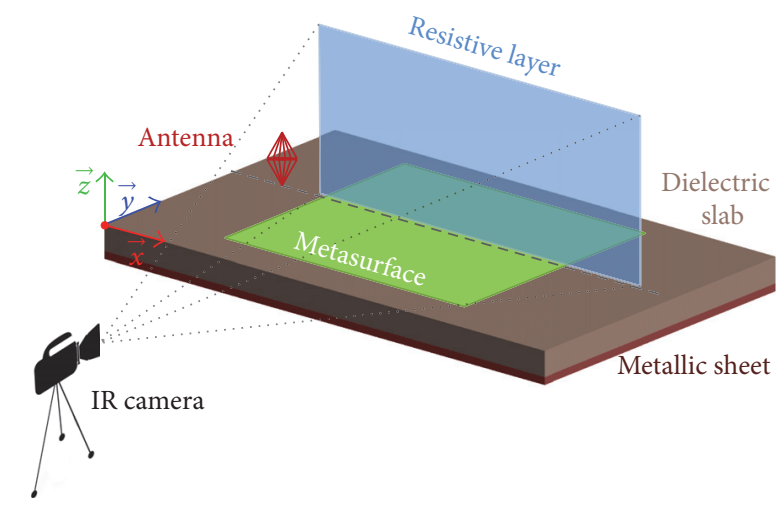

FIGURE 4: Measurement method based on visualization of electromagnetic field heating of a thin resistive layer.

SPP is a slow wave having nontilted equiphase planes $[22,26]$. As shown in the next section, SPP is a good candidate for focalization of energy above the interface, at least near the transmitting antennas, since the significant attenuation only occurs normally to the interface ( $z$ direction); the electric field is then oriented in the $z$ direction while the propagation occurs in the $x$ direction [32,34]. The metasurface used here is the basic brick of our Surface Wave launcher.

Nevertheless, a clear formal link appears in (1) between the field at the interface and Zenneck's tilted wave and those tilted waves might be observed when modifying some measurement parameters.

\section{Measurements}

3.1. Measurement Method. For obvious reasons, it is not easy to build the set-up in the HF band. Hence we perform the simulation in the HF band and conduct the experimentation in the UHF band, thanks to the scale principle.

The measurement method should characterize the field in the vicinity of the source without disturbing its distribution. That is the reason why we had carried out our measurement with the Electromagnetic and Infrared (EMIR) method. EMIR method consists in setting a thin resistive layer located where the field needs to be known and an infrared camera to image the film temperature (Figure 4). Since the resistive layer has a surface impedance close to the free space one, the local field is almost not disturbed and the temperature's increase is related to the tangential electric field strength [36]. In order to collect exclusively the temperature elevation due to the antenna radiated field, the transmitted UHF carrier is modulated with a very low frequency modulating signal. After demodulation an image which contains only the radiated electromagnetic field is obtained (Figure 4).

3.2. Results. Using a metamaterial, we succeed in visualizing SPP, as shown in Figure 5, and confined the energy above the interface [37].

Nevertheless, as mentioned before, a plasmon cannot be assimilated to a ZW. Indeed, the equiphase planes are not tilted and the phase speed is slower than the speed of light 


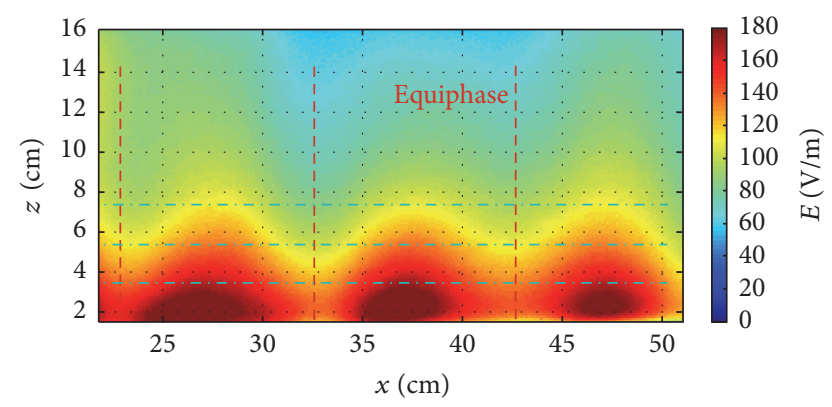

FIgURE 5: The SPP is observed with the EMIR method: equiphase planes are not tilted.

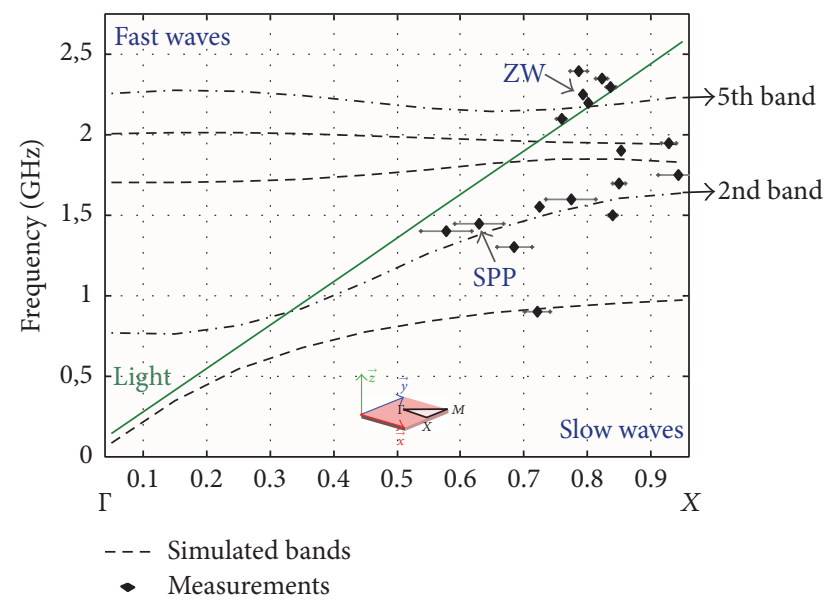

Figure 6: Dispersion diagram of the metasurface used for the experiment is drawn between $\Gamma$ and $X$.

although the ZW speed exceeds the speed of light. Therefore, a ZW might be observed if $\Re_{e}\left\{\sqrt{k_{0}^{2}-k_{z}^{2}}\right\}$ is less than $k_{0}$ when shifting the pole.

As shown in the dispersion diagram (Figure 6) over a dispersive medium such as a metamaterial, this condition may be reached at higher frequencies than in the case of SPP, notably just after a band gap. In Figure 6, we compare simulation results and measurement results. The metasurface is lying over a lossless grounded slab in the $\{\vec{x} ; \vec{y}\}$ plane, and the measurement has been done in the propagation plane from $0.8 \mathrm{GHz}$ up to $3 \mathrm{GHz}$ as shown in Figure 4. The light (black line) separates the diagram into two parts, the fast wave as the ZW (upper left) and the slow waves as the SPP (lower right). The simulations are performed with the eigenvalue mode solver of CST MWS ${ }^{\circledR}$ (dashed lines).

Before the band gap, in the 2nd band at $1.45 \mathrm{GHz}$, the Surface Plasmon focusses the energy just above the metamaterial (Figure 5). The SPP is well confined below $8 \mathrm{~cm}$ (0.6 wavelengths). In contrast, at $2.2 \mathrm{GHz}$, in the 5th band, a wave propagates obliquely (Figure 7 ) with a phase speed above the speed of the light. As seen in the literature and observed with simulation, the wave seems to sink towards the interface. To ensure that the observed propagation mode is not due to the grounded slab, a measurement is carried

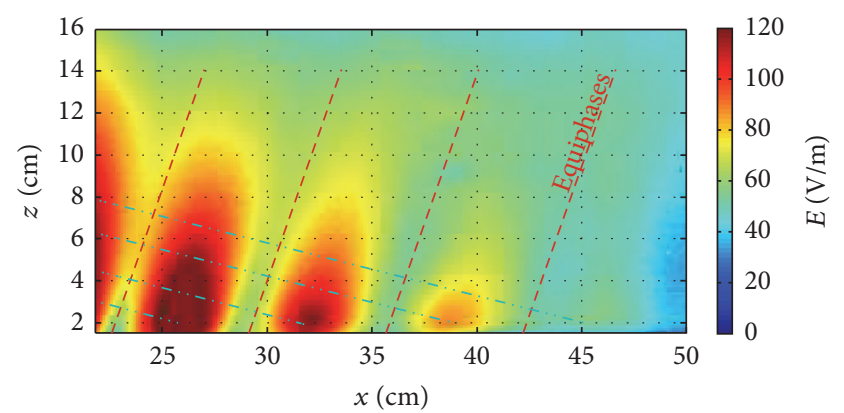

FIGURE 7: Measurement done at $2.2 \mathrm{GHz}$ with EMIR method; the wave seems to sink into the lower dielectric: equiphase planes are tilted.

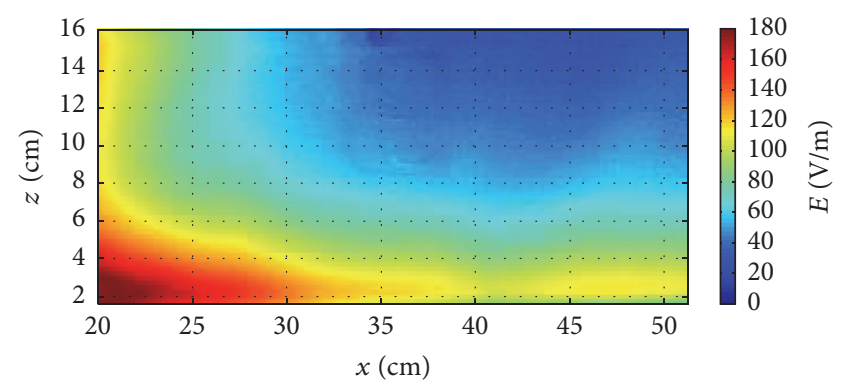

FIGURE 8: Field observed at $2.2 \mathrm{GHz}$ with the EMIR method when the metasurface is removed.

out without the metasurface. Without the dispersion of the metasurface we obtained a classical shape of field over an air/dielectric interface (Figure 8).

\section{Conclusion}

Our first objective was to enhance Surface Wave excitation for HF Surface Wave Radar. Studying the physical problem, it is clear that strong confinement of the energy near the interface can be obtained using negative permittivity materials. A Surface Plasmon Polariton is then generated and imaged by the EMIR method in the UHF instead of HF band for experimentation convenience. Such a metasurface can then be used to improve radiation at low elevation. Nevertheless, at upper frequency than the Surface Plasmon Polariton one, we observed a Zenneck-like structure of wave sinking toward the dielectric. This unusual observation is made thanks to the EMIR method which allows visualizing the electromagnetic field near the source and near the interface with a low impact on the field.

\section{Competing Interests}

The authors declare that there is no conflict of interests regarding the publication of this article. 


\section{References}

[1] J. Zenneck, "On propagation of electromagnetic waves along a flat planar conductor surface and its relation to wireless telegraphy," Annals of Physics, vol. 328, no. 10, pp. 846-866, 1907.

[2] C. A. Ziegler, "Technology and the process of scientific discovery: the case of cosmic rays," Technology and Culture, vol. 30, no. 4, pp. 939-963, 1989.

[3] A. Sommerfeld, "About the propagation of waves in wireless telegraphy," Annals of Physics, vol. 333, pp. 665-736, 1909.

[4] A. Sommerfeld, "About the propagation of waves in wireless telegraphy," Annalen der Physik, vol. 367, p. 96, 1920.

[5] J. R. Wait, "The ancient and modern history of EM groundwave propagation," IEEE Antennas and Propagation Magazine, vol. 40, no. 5, pp. 7-24, 1998.

[6] P. C. Clemmow, "Radio propagation over a flat earth across a boundary separating two different media," Philosophical Transactions of the Royal Society of London, Series A: Mathematical and Physical Sciences, vol. 246, pp. 1-55, 1953.

[7] T. K. Sarkar, W. Dyab, M. N. Abdallah et al., "Electromagnetic macro modeling of propagation in mobile wireless communication: theory and experiment," IEEE Antennas and Propagation Magazine, vol. 54, no. 6, pp. 17-43, 2012.

[8] D. A. Hill and J. R. Wait, "Ground wave attenuation function for a spherical earth with arbitrary surface impedance," Radio Science, vol. 15, no. 3, pp. 637-643, 1980.

[9] R. W. P. King and S. S. Sandler, "Electromagnetic field of a vertical electric dipole in the presence of a three-layered region," Radio Science, vol. 29, no. 1, pp. 97-113, 1994.

[10] G. Goubau, "On the Excitation of Surface Waves," Proceedings of the IRE, vol. 40, no. 7, pp. 865-868, 1952.

[11] A. F. Wickersham, "Generation, detection and propagation on the earth's surface of HF and VHF radio surface waves," Nature Physical Science, vol. 230, pp. 125-130, 1971.

[12] R. Paknys and D. R. Jackson, "The relation between creeping waves, leaky waves, and surface waves," IEEE Transactions on Antennas and Propagation, vol. 53, no. 3, pp. 898-907, 2005.

[13] D. A. Hill and J. R. Wait, "Excitation of the Zenneck surface wave by a vertical aperture," Radio Science, vol. 13, no. 6, pp. 969-977, 1978.

[14] J. Brown, "Some theoretical results for surface wave launchers," IRE Transactions on Antennas and Propagation, vol. 7, no. 5, pp. 169-174, 1959.

[15] T. Kahan and G. Eckart, "On the electromagnetic surface wave of sommerfeld," Physical Review, vol. 76, no. 3, pp. 406-410, 1949.

[16] A. V. Kukushkin, "A technique for solving the wave equation and prospects for physical applications arising therefrom," Physics-Uspekhi, vol. 36, no. 2, pp. 81-93, 1993.

[17] Y. B. Bashkuev, V. B. Khaptanov, and M. G. Dembelov, "Experimental proof of the existence of a surface electromagnetic wave," Technical Physics Letters, vol. 36, no. 2, pp. 136-139, 2010.

[18] A. V. Kukushkin, A. A. Rukhadze, and K. Z. Rukhadze, "On the existence conditions for a fast surface wave," Physics-Uspekhi, vol. 55, no. 11, pp. 1124-1133, 2012.

[19] R. T. Ling, J. D. Scholler, and P. Y. Ufimtsev, “The propagation and excitation of surface waves in an absorbing layer," Progress In Electromagnetics Research, vol. 19, pp. 49-91, 1998.

[20] A. Ishimaru, J. R. Thomas, and S. Jaruwatanadilok, "Electromagnetic waves over half-space metamaterials of arbitrary permittivity and permeability," IEEE Transactions on Antennas and Propagation, vol. 53, no. 3, pp. 915-921, 2005.
[21] H. M. Barlow and A. L. Cullen, "Surface waves," Proceedings of the IEE-Part III: Radio and Communication Engineering, vol. 100, no. 68, pp. 329-341, 1953.

[22] A. Ishimaru, Electromagnetic Wave Propagation, Radiation, and Scattering, Prentice-Hall, Englewood Cliffs, NJ, USA, 1990.

[23] K. A. Norton, "Propagation of radio waves over a plane earth," Nature, vol. 135, no. 3423, pp. 954-955, 1935.

[24] P. S. Epstein, "Radio-wave propagation and electromagnetic surface waves," Proceedings of the National Academy of Sciences of the United States of America, vol. 33, pp. 195-199, 1947.

[25] S. N. Karp and F. C. Karal, "Phenomenological theory of multimode surface wave excitation, propagation and diffraction," Tech. Rep. EM 198, New York University-Institute of Mathematical Sciences-Division of Electromagnetic Research, 1964.

[26] C. L. C. Smith, N. Stenger, A. Kristensen, N. A. Mortensen, and S. I. Bozhevolnyi, "Gap and channeled plasmons in tapered grooves: a review," Nanoscale, vol. 7, no. 21, pp. 9355-9386, 2015.

[27] L. Petrillo, F. Jangal, M. Darces, J.-L. Montmagnon, and M. Hélier, "Negative permittivity media able to propa-gate a surface wave," Progress in Electromagnetics Research, vol. 115, pp. 1-10, 2011.

[28] Y. V. Kistovich, "Possibility of observing Zenneck surface waves in radiation from a source with a small vertical aperture," Soviet Physics: Technical Physics, vol. 34, pp. 391-394, 1989.

[29] K. A. Norton, "The propagation of radio waves over the surface of the earth and in the upper atmosphere," Proceedings of the Institute of Radio Engineers, vol. 24, no. 10, pp. 1367-1387, 1936.

[30] V. N. Datsko and A. A. Kopylov, "On surface electromagnetic waves," Physics-Uspekhi, vol. 51, no. 1, pp. 101-102, 2008.

[31] A. Yu Nikitin, S. G. Rodrigo, F. J. García-Vidal, and L. MartínMoreno, "In the diffraction shadow: norton waves versus surface plasmon polaritons in the optical region," New Journal of Physics, vol. 11, Article ID 123020, 2009.

[32] J. B. Pendry, L. Martín-Moreno, and F. J. Garcia-Vidal, "Mimicking surface plasmons with structured surfaces," Science, vol. 305, no. 5685, pp. 847-848, 2004.

[33] S. A. Maier, Plasmonics: Fundamentals and Applications, Springer Science \& Business Media, 2007.

[34] M. J. Lockyear, A. P. Hibbins, and J. R. Sambles, "Microwave surface-plasmon-like modes on thin metamaterials," Physical Review Letters, vol. 102, no. 7, Article ID 073901, 2009.

[35] A. Ishimaru, Electromagnetic Wave Propagation, Radiation, and Scattering, Prentice Hall, Chapter 3 Section 11: Slow-Wave Solution for the TM Mode, Chapter 3 Section 12: Zenneck Waves and Plasmons, 1991.

[36] D. Balageas and P. Levesque, "EMIR: a photothermal tool for electromagnetic phenomena characterization," Revue Génrale de Thermique, vol. 37, no. 8, pp. 725-739, 1998.

[37] F. Jangal, L. Petrillo, M. Darces, M. Hélier, and J. Montmagnon, "Inductive surface element," WO2014029947, 2014. 


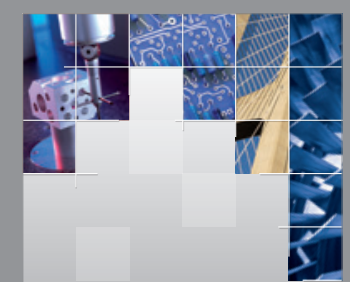

\section{Enfincering}
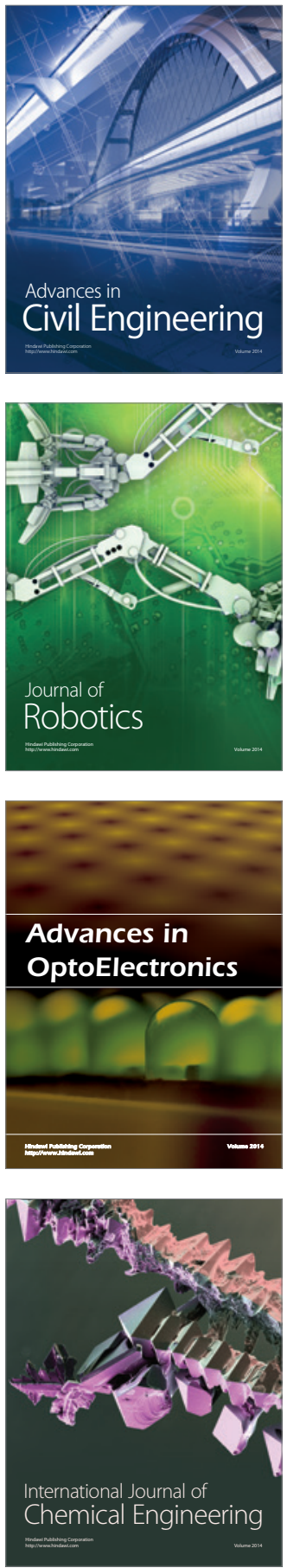

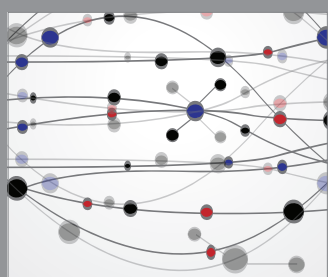

The Scientific World Journal

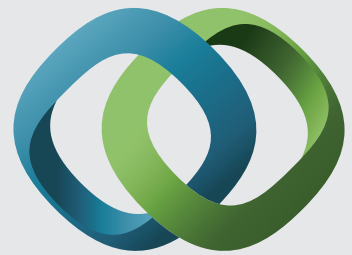

\section{Hindawi}

Submit your manuscripts at

http://www.hindawi.com
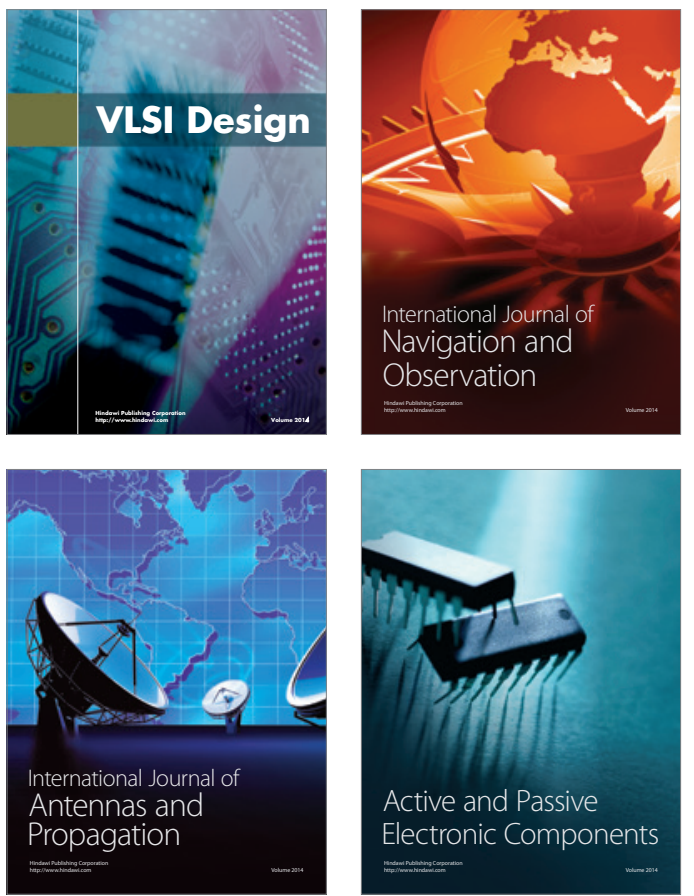
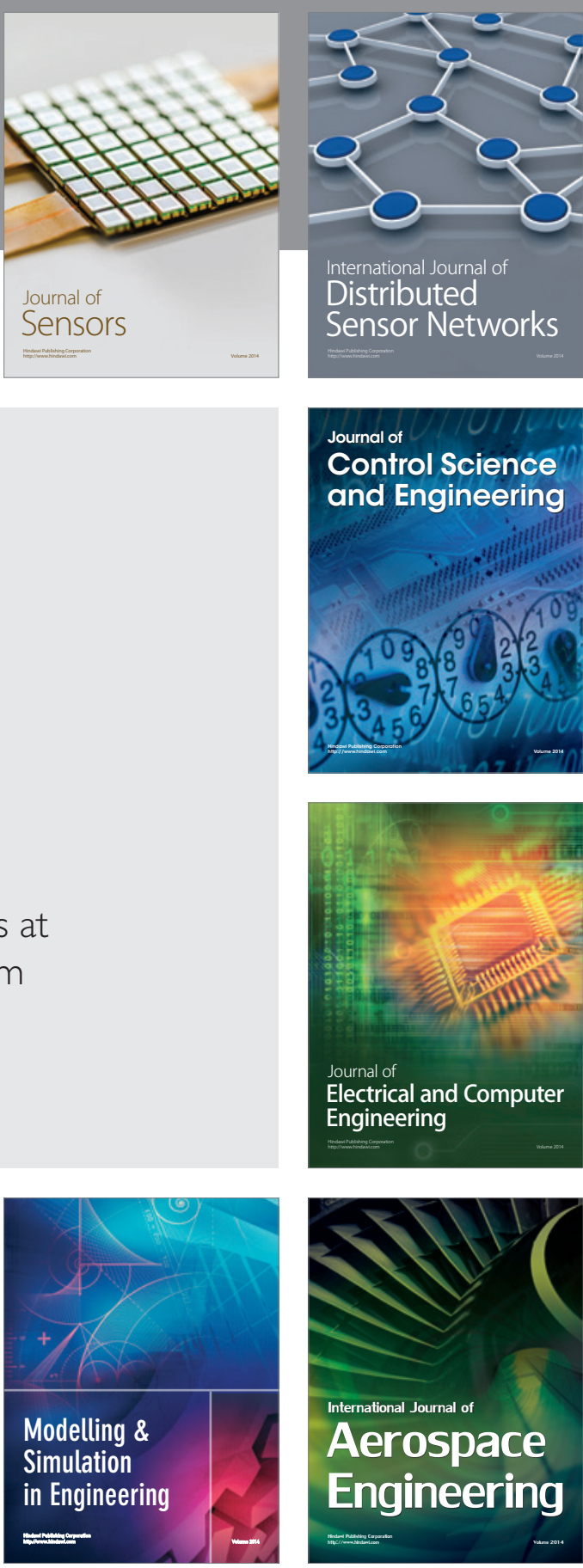

International Journal of

Distributed

Sensor Networks

Journal of

Control Science

and Engineering
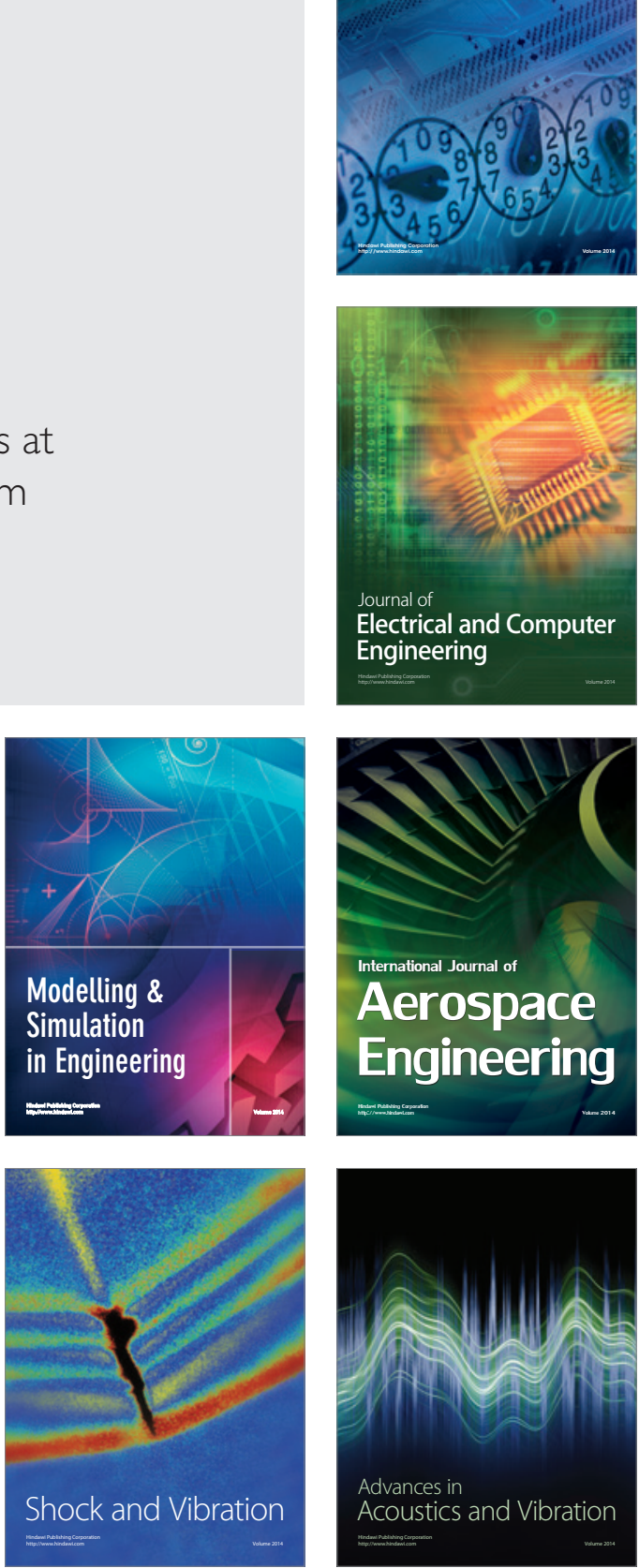